\title{
Primer registro fehaciente de Nopachtus coagmentatus (Xenarthra, Cingulata, Glyptodontidae) en la región Pampeana, Argentina. Contexto estratigráfico
}

\section{First reliable record of Nopachtus coagmentatus (Xenarthra, Cingulata, Glyptodontidae) in the Pampean region, Argentina. Stratigraphic context}

\author{
M. Zamorano ${ }^{1}$, M. De los Reyes ${ }^{1,2}$, D.G. Poiré ${ }^{3}$, G.J. Scillato-Yané ${ }^{1}$ \\ 1 División Paleontología de Vertebrados, Museo de La Plata, Facultad de Ciencias Naturales y Museo, Universidad Nacional \\ de La Plata, Paseo del Bosque s/n, 1900 La Plata, Argentina. CONICET. Email: marzamorano@fcnym.unlp.edu.ar \\ 2 Facultad de Ciencias Naturales y Museo de La Plata, Paseo del Bosque s/n (1900), La Plata, Buenos Aires, Argentina \\ 3 CIG de Investigaciones Geológicas, UNLP-CONICET, Calle 1 N$^{\circ} 644,1900$ La Plata, Argentina
}

\section{RESUMEN}

La especie Nopachtus coagmentatus fue reconocida por Ameghino en 1888, basándose en porciones del tubo caudal y la coraza dorsal; estos restos proceden de los Valles de las Sierras de Córdoba, en las cercanías de Villa Cura Brochero; y, estratigráficamente, de la Formación Brochero (Montehermosense - Chapadmalalense [Plioceno temprano - Plioceno tardío]). Ameghino también la menciona para la Formación Monte Hermoso (Montehermosense), pero la determinación es altamente dudosa. La explotación de rocas precámbricas en el núcleo central de las Sierras Bayas de Olavarría (provincia Buenos Aires) posibilitó el reconocimiento de la Formación El Polvorín (Plioceno) y, suprayacentes a esta, otras dos: Formación Esperanza y Formación El Búho (Pleistoceno tardío). El material que se da a conocer en esta comunicación fue exhumado en la Cantera Alcancía (3658'40".06 S; 60¹2'23".92 O), en niveles correspondientes al Chapadmalalense Superior de la Formación El Polvorín. El material mencionado consiste en osteodermos de la región dorso-lateral de la porción posterior de la coraza dorsal de $N$. coagmentatus. Se da a conocer el primer registro fehaciente de $N$. coagmentatus para la región Pampeana, se describe el material hallado, se detalla su procedencia y se presenta el perfil estratigráfico detallado correspondiente.

Palabras clave: Nopachtus coagmentatus; Chapadmalalense Superior; Plioceno; Olavarría; región Pampeana; Argentina.

\section{ABSTRACT}

The species Nopachtus coagmentatus was recognized by Amegino in 1888, on the basis of sections of the caudal tube and the dorsal carapace. These remains comes from Valles de las Sierras de Córdoba near Villa Cura Brochero, and stratigraphically from the Brochero Formation (Montehermosan-Chapadmalalan

Recibido el 18 de junio de 2014 / Aceptado el 5 de diciembre de 2014 / Publicado online el 17 de abril de 2015

Citation / Cómo citar este artículo: Zamorano, M. et al. (2015). Primer registro fehaciente de Nopachtus coagmentatus (Xenarthra, Cingulata, Glyptodontidae) en la región Pampeana, Argentina. Contexto estratigráfico. Estudios Geológicos 71(1): e027. http://dx.doi. org/10.3989/egeol.41808.339.

Copyright: (C) 2015 CSIC. This is an open-access article distributed under the terms of the Creative Commons Attribution-Non Commercial (by-nc) Spain 3.0 License. 
[early Pliocene - late Pliocene]). Ameghino also mentioned N. coagmentatus for the Monte Hermoso Formation (Montehermosan), but the determination Ameghino is highly questionable. The exploitation of Precambrian rocks from the center of the Sierras Bayas in Olavarría (Buenos Aires province) allowed the identification from the bottom to the top, of La Alcancía Formation (Miocene), El Polvorín Formation (Pliocene), La Esperanza Formation and El Búho Formation (both late Pleistocene). In this contribution present osteoderms of the latero-dorsal portion of the posterior region of the dorsal carapace of $N$. coagmentatus. The specimen was extracted from brownish sandy-silt sediments of La Alcancía Quarry (36 ${ }^{\circ} 58^{\prime} 40^{\prime \prime} .06 \mathrm{~S} ; 60^{\circ} 12^{\prime 2} 3^{\prime \prime} .92 \mathrm{~W}$ ) corresponding to El Polvorín Formation (Upper Chapadmalalan) and correspond to the first reliable record of $N$. coagmentatus for the Pampean region. Finally, we offer a detailed description of the specimen, and its geographic and stratigraphic provenance.

Key words: Nopachtus coagmentatus; Upper Chapadmalalan; Pliocene; Olavarría; Pampeana region; Argentina.

\section{Introducción}

Los gliptodontes constituyen un grupo de Cingulata (Xenarthra), muchos de tamaño gigantesco (Scillato-Yané \& Carlini, 1998; Fariña, 2001; Soibelzon et al., 2012). Hoffstetter (1958) dividió a los gliptodóntidos en cinco subfamilias: 1) Glyptatelinae; 2) Propalaehoplophorinae; 3) Glyptodontinae; 4) Doedicurinae; y 5) Hoplophorinae (=Sclerocalyptinae). A su vez, a esta última la subdividió en siete tribus: a) Palaehoplophorini; b) Neuyurini; c) Neothoracophorini; d) Plohophorini; e) Sclerocalyptini; f) Lomaphorini; y g) Panochthini. Castellanos (1927) fue el primero en considerar como un grupo taxonómico los géneros, Nopachthus (sic) Ameghino, 1888a, Propanochthus Castellanos, 1925 y Panochthus Burmeister, 1866. En esa oportunidad le otorga a dicho agrupamiento la categoría taxonómica de subfamilia y le da el nombre de Panochthinae. Simpson (1945) le confiere a este grupo de tres géneros (Nopachtus, Propanochthus y Panochthus) la categoría taxonómica de tribu (Tribu Panochthini). Ameghino (1888a) fundó el género Nopachtus para incluir $N$. coagmentatus, especie que reconoció basándose en porciones del tubo caudal y la coraza dorsal; estos restos proceden de los Valles de las Sierras de Córdoba, en las cercanías de Villa Cura Brochero; y, estratigráficamente, del "Brocherense", sensu Castellanos, 1942.

Posteriormente, Castellanos (1925) añadió a este género Nopachtus trouessarti (Moreno, 1888) (originalmente incluida en Panochthus), una especie reconocida a partir de un fragmento de la región postero-dorsal izquierda de la coraza dorsal, con cinco hileras transversales de osteodermos, procedente según este autor de "Monte Hermoso", provincia de Buenos Aires, sin dar más detalles (Moreno, 1888). Zamorano et al. (2011) transfieren $N$. trouessarti a Phlyctaenopyga Cabrera, 1944 y reconocen N. cabrerai Zamorano, Scillato-Yané, González
Ruiz \& Zurita, 2011. De esta manera, Nopachtus queda constituido por dos especies: $N$. coagmentatus y N. cabrerai (ver Zamorano et al., 2011; Zamorano, 2012a, 2012b).

El holotipo de la especie tipo del género (N. coagmentatus) se registra para la Formación Brochero (Montehermosense - Chapadmalalense [Plioceno temprano - Plioceno tardío] [ver Cruz, 2013]) de las Sierras de Córdoba, en las cercanías de Villa Cura Brochero, provincia de Córdoba.

Ameghino (1888b, p. 479-480) asigna a N. coagmentatus "... un trozo considerable de coraza", de Monte Hermoso, pero no justifica dicha asignación, pues no lo describe ni figura; tampoco indica dónde está depositado. Sumado esto a la circunstancia de que Ameghino obviamente desconocía a N. cabrerai, la mención de $N$. coagmentatus en Monte Hermoso por parte de Ameghino resulta sumamente dudosa.

A su vez, N. cabrerai se registra en la Formación Monte Hermoso (Montehermosense, Plioceno temprano) del SO de la provincia de Buenos Aires (Zamorano et al., 2011; Zamorano, 2012a, 2012b).

En esta contribución se presenta el primer registro fehaciente de Nopachtus coagmentatus para la región Pampeana. Asimismo, se describe el perfil estratigráfico del lugar de hallazgo, el cual constituye un nuevo aporte a la geología del centro de la región Pampeana de la Argentina.

\section{Procedencia geográfica y estratigráfica general}

El Neógeno/Cuaternario del área intraserrana de la región nororiental del Sistema de Tandilia, en la Cantera Alcancía (36 $58^{\prime} 40^{\prime \prime} .06 \mathrm{~S} ; 60^{\circ} 12^{\prime} 23^{\prime} .92$ O), Olavarría (Fig. 1), presenta de base a techo las siguientes unidades estratigráficas (Poiré, 2012): Formaciones La Alcancía, El Polvorín, La Esperanza y El Búho. Este conjunto sedimentario se apoya 


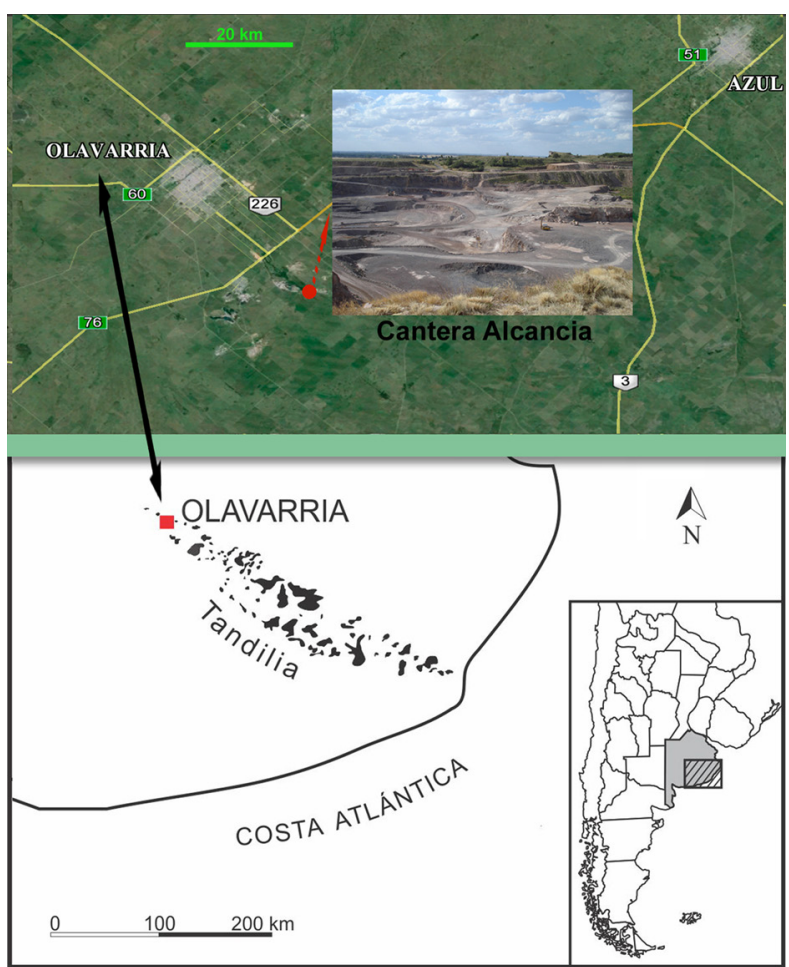

Fig. 1.—Ubicación geográfica de la Cantera Alcancía.

sobre el Neoproterozoico del Grupo Sierras Bayas y Formación Cerro Negro (Poiré \& Gaucher, 2009).

La Formación La Alcancía (Fig. 2) presenta hasta cuatro metros de espesor de niveles conglomerádicos gruesos asociados a niveles pelíticos rosados y calcretes mamelonares extremadamente blanquecinos (Poiré, 2012).

La Formación El Polvorín (Pleistoceno tardíoHoloceno temprano) está constituida por: a) brecha y conglomerado (facies de psefita), y b) arenisca fina y fangolita (facies loéssica). La primera se distribuye en un nivel basal espeso, de hasta cuatro metros de potencia, y lentes de menor tamaño en el seno del material loéssico (Fig. 2). La facies loéssica se dispone por arriba del cuerpo de psefita basal, con un espesor de hasta siete metros. Además de los osteodermos aquí analizados, de estas facies se ha exhumado una fauna correspondiente al Chapadmalalense Superior (De los Reyes et al., 2013, Zurita et al., 2014) coincidiendo con la hipótesis planteada por Deschamps et al. (2012).

Por arriba de la Formación El Polvorín, se dispone en contacto erosivo la Formación La Esperanza (Poiré, 2012), la cual consta de sedimentita limoarenosa de color castaño de hasta seis metros de potencia, de moderada compactación, con abundantes niveles horizontales de entoscamiento. Presenta cárcavas y paleocuevas, y abundantes restos fósiles, con una pobre mineralización y carbonato adherido, asignables por la presencia de Equidae Equus (A.) neogaeus Lund, 1840 al Piso/Edad Lujanense (Pleistoceno tardío-Holoceno temprano).

En tope de la columna se encuentra la Formación El Búho (Poiré, 2012), la cual presenta una fuerte discordancia erosiva en su base, que incluso llega afectar hasta la sedimentita de la Formación El Polvorín, se desarrolla la depositación de arenisca fina castaña, loésica, de hasta cinco metros de espesor, en parte friable, de buena selección, y masivas. La fauna exhumada es corresponde al Piso/Edad Lujanense. Estas unidades sedimentarias presentan fuertes superficies erosivas dado que se encuentran enmarcadas en un área eminentemente serrana, con signos de levantamiento tectónico concomitante (Poiré et al., 2005, Poiré, 2012).

\section{Materiales y métodos}

Se intentó determinar la ubicación aproximada de los osteodermos de Nopachtus coagmentatus aquí presentados (Xen-45) en las distintas regiones de la coraza dorsal. A tal efecto, se los comparó con aquellos de la coraza dorsal más completa existente de $N$. coagmentatus (holotipo: MLP 16-122).

El esquema bioestratigráfico utilizado corresponde al de Cione \& Tonni (1999, 2005).

Abreviaturas utilizadas: MLP, Museo de la Plata, División Paleontología Vertebrados, Facultad de Ciencias Naturales y Museo, Universidad Nacional de La Plata, Buenos Aires, Argentina; Xen-45: colección Cementos Avellaneda, Olavarría Buenos Aires, Argentina.

\section{Paleontología sistemática}

Magnorden XENARTHRA Cope, 1889

Orden CINGULATA Illiger, 1811

Suborden GLYPTODONTIA Ameghino, 1889

Superfamilia GLYPTODONTOIDEA Gray, 1869

Familia GLYPTODONTIDAE Gray, 1869

Género Nopachtus Ameghino, 1888a 


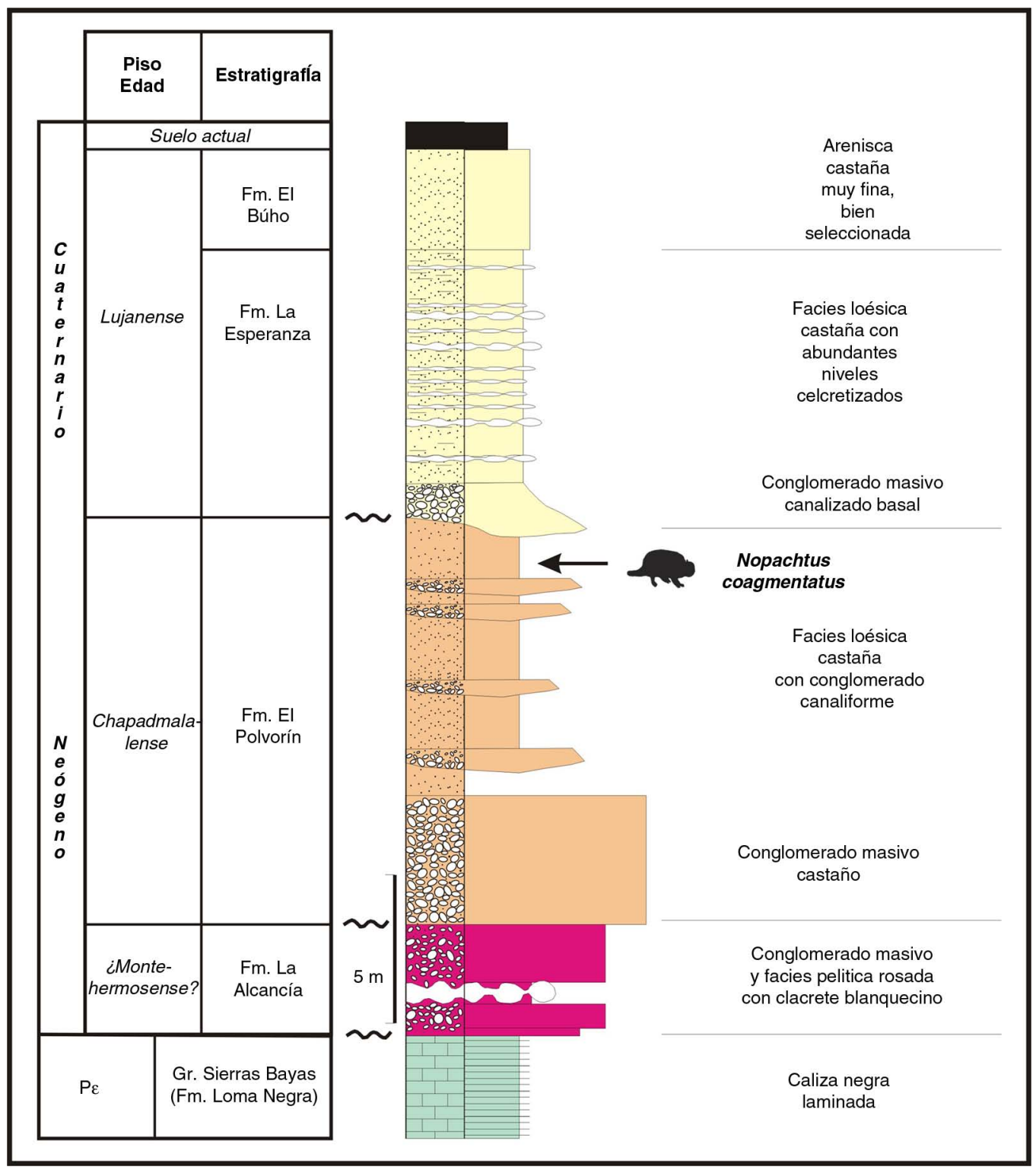

Fig. 2.-Perfil geológico de la Cantera Alcancía.

Especie tipo: Nopachtus coagmentatus Ameghino, $1888 \mathrm{a}$, por designación original. Formación Brochero (Montehermosense - Chapadmalalense [Plioceno temprano -Plioceno tardío]) de las Sierras de Córdoba.

\section{Nopachtus coagmentatus Ameghino, 1888}

Material examinado: Xen-45, fragmentos de la región dorso-lateral de la mitad posterior de la coraza dorsal, los cuales comprenden de uno a cinco osteodermos (no todos completos) (Fig. 3B).
Procedencia estratigráfica y geográfica del material examinado: Facies loéssica de la Formación El Polvorín (Chapadmalalense Superior, Plioceno) (Fig. 2) de las Sierras Bayas de Olavarría (provincia de Buenos Aires) (Fig. 1).

\section{Descripción:}

Cabe señalar que, hasta el momento, en los gliptodóntidos no es posible identificar a qué lado de la coraza (derecha/izquierda) pertenecen osteodermos como los aquí presentados; esta identificación 
(a)

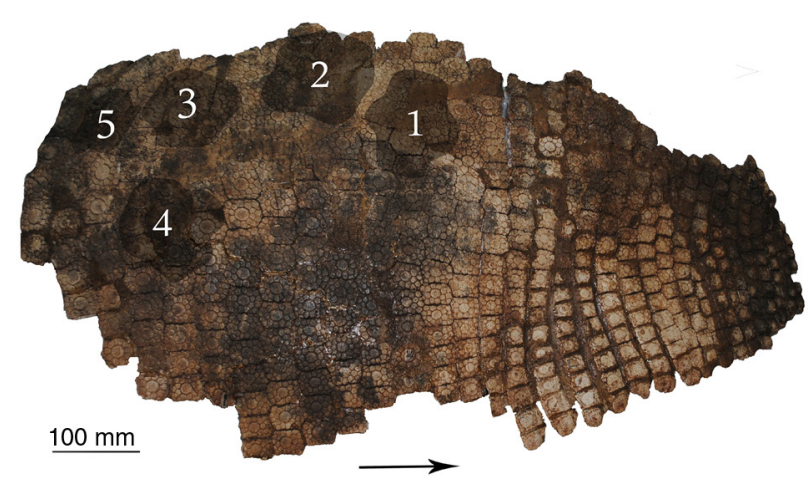

(b)

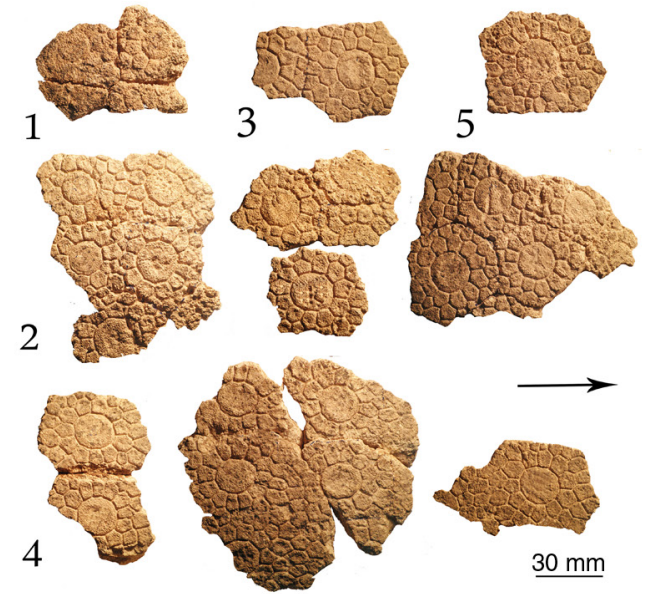

Fig. 3.-Nopachtus coagmentatus A_ MLP 16-22 (holotipo), porción lateral derecha de la coraza dorsal (Montehermosense de Córdoba). B_Xen-45, fragmentos de la coraza dorsal (Chapadmalense Superior de la Provincia de Buenos Aires); los números indican la región de la coraza a la que corresponden. Las flechas señalan la parte anterior de la coraza.

sí se puede hacer en dasipódidos (Scillato-Yané, 1975, 1982).

Los osteodermos presentan una figura central rodeada por dos hileras de figuritas periféricas; en los osteodermos de la región más anterior de la coraza, solo por una. En la mitad posterior de la coraza, la primera hilera incluye entre 10 y 12 figuritas periféricas (13, con una figurita muy reducida, en un solo osteodermo) pentagonales o hexagonales, como en $N$. cabrerai. A su vez, la primera hilera está rodeada por una segunda hilera incompleta de figuritas periféricas. En los casos en los cuales fue posible contarlas, se hallaron dos osteodermos con 19 figuritas, otros dos con 20, tres con 21 y uno con 22 .

En la mitad posterior de la coraza de $N$. coagmentatus, la segunda hilera tiene más figuritas que la de Plohophorus figuratus Ameghino, 1887 (16 a 17, según Ameghino, 1889) y Pseudohoplophorus absolutus Perea, 2005 (dos figuritas, solo en algunas placas de la zona lateral, ver Perea, 2005), pero menos que en Phlyctaenopyga ameghini Ameghino, 1889 (de 23 a 27, ver Zamorano et al., 2011) y en Stromaphorus compressidens (Moreno y Mercerat, 1891) (entre 18 y 21, ver Cabrera, 1944, fig. 18).

Las figuras centrales son de casi planas a levemente deprimidas, en tanto que en la misma región de la coraza dorsal de $N$. cabrerai son de elevadas a francamente convexas, con aspecto de ampollas.

\section{Discusión}

De acuerdo con los análisis filogenéticos más recientes, Hoplophorinae no es un grupo natural (Fariña, 1993; Fernicola, 2005, 2008; Porpino et al., 2009, 2010; Fernicola \& Porpino, 2012) y tampoco lo es Panochthini (Zamorano, 2012a, 2012b; Zamorano \& Brandoni, 2013). Zamorano (2012a, 2012b) y Zamorano \& Brandoni (2013), a base de análisis filogenéticos, relacionan en un clado, Nopachtus coagmentatus, N. cabrerai, Propanochthus bullifer (ver Zamorano, 2013), Stromaphorus compressidens y Phlyctaenopyga ameghini. En el apartado "Paleontología sistemática" se coloca el género Nopachtus inmediatamente debajo de la Familia Glyptodontidae y no se anota ni la Subfamilia "Hoplophorinae" Huxley, 1864, ni la Tribu "Panochthini" Castellanos, 1927.

Entre los fósiles colectados en la Formación El Polvorín de la Cantera Alcancia, además del material de $N$. coagmentatus presentado, en esos niveles se hallaron los siguientes taxones: Eosclerocalyptus sp. [Cingulata], Hegetotheria sp. [Notoungulata], Radiodiscus sp. [Charopidae, Gastropoda]. La presencia de estos taxones permite postular que los niveles portadores se corresponden con ambientes abiertos (Bond et al., 1995; Scillato-Yané et al., 1995; Miguel \& Aguirre, 2011). 


\section{Conclusiones}

El material presentado es atribuible a $N$. coagmentatus por los siguientes caracteres: los osteodermos presentan una figura central rodeada por dos hileras de figuritas periféricas, solo por una en la región más anterior de la coraza; la primera hilera incluye entre 10 y 12 figuritas periféricas pentagonales o hexagonales; si hay una segunda hilera tiene entre 19 y 22 figuritas.

$N$. coagmentatus, hasta la presente contribución, se registraba únicamente para la Formación Brochero (Montehermosense - Chapadmalalense [Plioceno temprano - Plioceno tardío]) de las Sierras de Córdoba. A partir de este nuevo hallazgo se amplía la distribución estratigráfica y geográfica de la especie, la cual ahora se registra para el Chapadmalalense Superior de las Sierras Bayas de Olavarría. Por primera vez se describe material de Nopachtus coagmentatus hallado en la región Pampeana. El nivel del cual proviene el material de $N$. coagmentatus corresponde a ambientes serranos y abiertos del Plioceno.

\section{AGRADECIMIENTOS}

A Sergio E. Miguel, por la buena predisposición para identificar el gastropoda. A Juan Cruz González, por las fotografías y la edición de estas. A la empresa Cementos Avellaneda S.A. A la Familia Pezzuchi. A los revisores, por enriquecer el trabajo. A Marcelo Reguero, por la manipulación del material tipo. A Laureano R. González Ruiz y Noelia L. Illarreguy, por colaborar en la redacción del abstract.

\section{Referencias}

Ameghino, F. (1887). Apuntes preliminares sobre algunos mamíferos extinguidos del yacimiento de "Monte Hermoso" existentes en el "Museo La Plata". Boletín del Museo La Plata, 1: 1-20.

Ameghino, F. (1888a). Rápidas diagnosis de algunos mamíferos fósiles nuevos de la República Argentina. In: A.J. Torcelli (Ed.) Obras Completas y Correspondencia Científica de Florentino Ameghino. vol. 5. Taller de Impresiones Oficiales, p. 471-480.

Ameghino, F. (1888b). Lista de las especies de mamíferos fósiles del mioceno superior de Monte Hermoso, hasta ahora conocidas: Buenos Aires, P.E. Coni, 21 pp.

Ameghino, F. (1889). Contribución al conocimiento de los mamíferos fósiles de la República Argentina. Actas Academia Nacional de Ciencias de Córdoba, 6: 1-1028.

Bond, M.; Cerdeño, E. \& López, G. (1995). Los ungulados nativos de América del Sur. In: Evolución biológica y climática de la región Pampeana durante los últimos cinco millones de años. Un ensayo de correlación con el Mediterráneo Occidental (Alberdi, M.T.; Tonni, E.P. \& Leone, G., Eds), España, Monografías de la CSIC, 257-275.

Burmeister, G. (1866). Lista de los mamíferos fósiles del terreno diluviano. Anales del Museo Público de Buenos Aires, 1: 121-232.

Cabrera, A. (1944). Los Gliptodontoideos del Araucaniano de Catamarca. Revista del Museo de La Plata (N. Serie), Paleontología 3: 5-76.

Castellanos, A. (1925). Breves notas sobre evolución de la coraza y del extremo caudal en los Loricata (=Hicanodonta). Revista de "El Circulo", número extraordinario, 93-96.

Castellanos, A. (1927). Descripción de un fragmento de tubo caudal de un nuevo daedicurino en relación con sus géneros afines. Anales del Museo de Historia Natural de Montevideo, 2: 265-300.

Castellanos, A. (1942). A propósito de los géneros Plohophorus, Nopachthus y Panochthus (3a. parte). Publicaciones del Instituto de Fisiografía y Geología de la Universidad Nacional del Litoral, 11: 413-592.

Cione, A.L. \& Tonni, E.P. (1999). Biostratigraphy and chronological scale of uppermost Cenozoic in the Pampean area, Argentina. In: Quaternary of South America and Antarctic Peninsula (Rabassa, J. \& Salemme, M.C., Eds.), 3: 23-52.

Cione, A.L. \& Tonni, E.P. (2005). Bioestratigrafía basada en mamíferos del Cenozoico Superior de la Provincia de Buenos Aires, Argentina. In: Relatorio del XVI Congreso Geológico Argentino (de Barrio, R.E.; Etcheverry, R.O.; Caballé, M.F. \& Llambías, E., Eds.), La Plata, 183-200.

Cope, E.D. (1889). The edentata of North America. American Naturalist, 23: 657-664.

Cruz, L.E. (2013). Biostratigraphy and geochronology of the late Cenozoic of Córdoba Province (central Argentina). Journal of South American Earth Sciences, 42: 250-259. http://dx.doi.org/10.1016/j. jsames.2012.06.015.

De los Reyes, M.; Poiré, D.G.; Soibelzon, L.; Zurita, A.E. \& Arouy, M.J. (2013). First evidence of scavenging in a Glyptodont (Mammalia, Glyptodontidae) from the Pliocene of the Pampean region (Argentina). Taphonomic and paleoecological remarks. Paleontologia electrónica, 16 (2)15A, 13.

Deschamps, C.A.; Vucetich, M.G.; Verzi, D.H. \& Olivares, A.I. (2012). Biostratigraphy and correlation of the Monte Hermoso Formation (early Pliocene, Argentina): The evidence from caviomorph rodents. Journal of South American Earth Sciences, 35: 1-9. http://dx.doi.org/10.1016/j.jsames.2011.10.006.

Fariña, R.A. (1993). Análisis filogenético de Glyptodontia. Ameghiniana, 30: 329-330.

Fariña, R.A. (2001). Física y Matemáticas para reconstruir la vida en el pasado. Actas de Fisiología, 6: 45-70. 
Fernicola, J.C. (2005). Análisis filogenético de la familia Glyptodontidae Gray: Ameghiniana, 42: 27.

Fernicola, J.C. (2008). Nuevos aportes para la sistemática de los Glyptodontia Ameghino 1889 (Mammalia, Xenarthra, Cingulata). Ameghinana, 45: 553-574.

Fernicola, J.C. \& Porpino, K.O. (2012). Exoskeleton and Systematics: A Historical Problem in the Classification of Glyptodonts. Journal of Mammalian Evolution, 19: 1-13. http://dx.doi.org/10.1007/ s10914-012-9186-1.

Gray, J.E. (1869). Catalogue of carnivorous, pachydermatous and edentate Mammalia in the British Museum. London, British Museum (Natural History), VII $+398 \mathrm{pp}$.

Hoffstetter, R. (1958). Xenarthra. In: Traité de Paléontologie (Piveteau, J., Ed.), París, Masson \& Cie., 6: 535-636.

Illiger, K. (1811). Prodromus Systematics Mammalium et Ayium. Berlin, Salfeld, XVIII+1-301 pp.

Lund, P.W. (1840). Nouvelles recherches sur la faune fossile du Brésil. Annales des Sciences Naturelles, 13: 310-319.

Miquel, S.E. \& Aguirre, M.L. (2011). Taxonomía de los gasterópodos terrestres del Cuaternario de Argentina. Revista Española de Paleontología, 26 (2): 101-133.

Moreno, F.P. (1888). Informe preliminar de los progresos del Museo La Plata durante el primer semestre de 1888, presentado al señor ministro de Obras Públicas de la provincia de Buenos Aires. Boletín del Museo La Plata, 1-35.

Moreno, F.P. \& Mercerat, A. (1891). Exploración arqueológica de la provincia de Catamarca: Paleontología. Revista del Museo de La Plata, 1: 222-236.

Perea, D. (2005). Pseudoplohophorus absolutus n. sp. (Xenarthra, Glyptodontidae), variabilidad en Sclerocalyptinae y redefinición de una biozona del Mioceno Superior de Uruguay. Ameghiniana, 42: 175-190.

Poiré, D.G. (2012). Secuencias sedimentaria Neoproterozoico-Paleozoico inferior del Sistema de Tandilia y su cobertura del Terciario superiorCuaternario en la zona de Olavarría. Guía de Campo. Centro de investigaciones Geológicas, 47.

Poiré, D.G.; Canessa, N.D.; Scillato-Yané, G.J.; Carlini, A.A.; Canalicchio, J.M. \& Tonni, E.P. (2005). La Formación El Polvorín: una nueva unidad del Neógeno de Sierras Bayas, Sistema de Tandilia, Argentina. XVI Congreso Geológico E.P. Argentino, Actas 1: 315-322.

Poiré, D.G. \& Gaucher, C. (2009). Lithostratigraphy. Neoproterozoic-Cambrian evolution of the Rio de la Plata Palaeocontinent. In: Neoproterozoic-Cambrian tectonics, global change and evolution: a focus on southwestern Gondwana. Developments in Precambrian Geology (Gaucher, C.; Sial, A.N.; Halverson, G.P. \& Frimmel, H.E., Eds.), Elsevier, 4: 87-101.
Porpino, K.O.; Fernícola, J.C. \& Bergqvist, L.P. (2010). Revisting the intertropical brazilian species Hoplophorus euphractus (Cingulata, Glyptodontoidea) and the phhylogenetic affinities of Hoplophorus. Journal of Vertebrate Paleontology, 30: 911-927. http://dx. doi.org/10.1080/02724631003765735.

Scillato-Yané, G.J. (1975). Nuevo géne La Plata ro de Dasypodidae (Edentata, Xenarthra) del Plioceno de Catamarca (Argentina). Algunas consideraciones filogenéticas y zoogeográficas sobre los Euphractini. I Congreso Argentino de Paleontología y Bioestratigrafía, Actas 2: 449-461.

Scillato-Yané, G.J. (1982). Los Dasypodidae (Mammalia, Edentata) del Plioceno y Pleistoceno de Argentina. Tesis Doctoral, Universidad Nacional de La Plata, Facultad de Ciencias Naturales y Museo, 159 pp.

Scillato-Yané, G.J.; Carlini, A.A.; Vizcaíno, S.F. \& OrtizJaureguizar, E. (1995). Xenarthra. In: Evolución biológica y climática de la región Pampeana durante los últimos cinco millones de años. Un ensayo de correlación con el Mediterráneo Occidental (Alberdi, M.T.; Tonni, E.P. \& Leone, G., Eds), España, Monografías de la CSIC, 183-209.

Simpson, G.G. (1945). The principles of classification and a classification of mammals. Bulletin American Museum of Natural History, 85: 1-350.

Zamorano, M. (2012a). Los Panochthini (Xenarthra, Glyptodontidae): Sistemática y evolución. Tesis Doctoral, Universidad Nacional de La Plata, Facultad de Ciencias Naturales y Museo, 278 pp.

Zamorano, M. (2012b). Los Panochthini (Xenarthra, Glyptodontidae): Sistemática y evolución. Mastozoología Neotropical, 19: 382-384.

Zamorano, M. (2013). Diagnosis y nueva descripción de Propanochthus bullifer (Burmeister) (Xenarthra, Glyptodontidae). Consideraciones bioestratigráficas y cronológicas de su procedencia. Spanish Journal of Palaeontology, 28(2): 283-292.

Zamorano, M. \& Brandoni, D. (2013). Phylogenetic analysis of the Panochthini (Xenarthra, Glyptodontidae, "Holplophorinae"). Alcheringa, 37 (4): 442-451. http://dx. doi.org/10.1080/03115518.2013.770224.

Zamorano, M.; Scillato-Yané, G.J.; González Ruiz, L.R. \& Zurita, A.E. (2011). Revisión de los géneros Nopachtus Ameghino y Phlyctaenopyga Cabrera (Xenarthra, Glyptodontidae, Hoplophorinae) del Mioceno tardío y Plioceno de Argentina. Revista del Museo Argentino de Ciencias Naturales "Bernardino Rivadavia”, 13: 59-68.

Zurita, A.E.; Taglioretti, M.; De los Reyes, M.; Oliva, C. \& Scaglia, F. (2014). First Neogene skulls of Doedicurinae (Xenarthra, Glyptodontidae): morphology and phylogenetic implications. Historical Biology. http:// dx.doi.org/10.1080/08912963.2014.969254. 